\title{
Slot Allocation Algorithm for Real Time and None Real Time Traffics of Mobile WiMAX IEEE802.16e
}

\author{
D. S. Shu'aibu \\ Faculty of Electrical Engineering \\ Universiti Teknologi Malaysia \\ Skudai Johor Bahru Malaysia
}

\author{
S. K. Syed Yusof \\ Faculty of Electrical Engineering \\ Universiti Teknologi Malaysia \\ Skudai Johor Bahru Malaysia
}

\begin{abstract}
In recent advancement in communication, it was made possible that many heterogeneous wireless networks can be made to operate in one wireless network by establishing standard for each wireless traffic. Slots allocation in such network is one of the challenges since each traffic has its own quality of service requirements. In this paper we present slot allocation algorithm for real time and none real time traffic of mobile WiMAX. Since the wireless link of the network is a function of time, the allocation of the slot is based on the condition of the wireless link. Hence we estimate the wireless link based on the total path loss and the SNR value of the packet to be scheduled. The simulation shows that the proposed algorithm has achieved higher throughput.
\end{abstract}

Keywords-service flow, buffer, scheduler, arrival time departure time, frame, deadline and latency

\section{INTRODUCTION}

WiMAX (IEEE802.16) provides service to many devices like notebook personal computer; handset, smart phone and some consumers' electronics such as gaming devices, cameras, camcorder, and music player [1]. It is the first of all IP mobile internet solution enabling efficient and scalable networks for data, video and voice $[1,2]$. It is one of the wireless metropolitan area networking that operates at scalable bandwidth, with different mode of access (OFDM and OFDMA) and also it uses different value of cyclic prefix [1], [2]. It supports QoS on the wireless domain and interfaces for ATM, IP, E1/T1 and Ethernet. Due to its functionality, it supports several broadband services like voice over IP and video on demand [2].

The IEEE802.16 standard defined five scheduling classes these are;- unsolicited grant service (UGS), real time service polling (rtPs), Extended real time service polling (ertPs), none real time polling service (nrtPs) and best effort (BE), but it does not provide information about the standard algorithms that should be used to provide QoS to the service flows (SFs). Call admission control and scheduling are some of algorithm that control the QoS when properly design. The call admission control has important role to play especially when combined with scheduling service, since they are meant to manage and guarantee the QoS requirements. Both call admission control (CAC) algorithm and scheduling service when carefully implemented provides a better QoS compare to implementing one of them alone [3]. In most cases, the two algorithms were designed separately; nevertheless each has to be efficient in managing the network resources. The CAC handles the link bandwidth while the scheduling handles the packet delay for slot allocation.
Recent research works have been conducted in order to provide QoS in IEEE 802.16 networks [3] - [8]. In [3], QoS bandwidth allocation in IEEE802.16 networks was presented. It is based on partition bandwidth allocation in the CAC and service flows were classified and accepted based on the availability of bandwidth on a particular partition. The bandwidth grant was based on grant per subscriber station (GPSS) mode; it was in the downlink, how packets should be scheduled was not presented in the paper. In [4], grant per connection (GPC) mode for bandwidth allocation is adopted for an uplink scheduling of IEEE802.16 and DOCSIS to support diverse QoS. Its centralized approach i.e. Point to multipoint mode wherein all scheduling decision is achieved through the BS. Nevertheless no simulation results were presented and the effectiveness of the architecture is not emphasized.

In [5], adaptive bandwidth allocation algorithms was presented, the algorithm propose was hierarchical structure for bandwidth allocation in the downlink which decide whether QoS for a particular connection can be satisfied at the base station or not, followed by a scheduling algorithm. To improve QoS in [5], [6] presented a QoS Bandwidth allocation to account for packet delay. The architecture is called QoS CAC and grant per connection mode (GPC) is adopted. Algorithm developed in [7], scheduled different traffics with information on individual packet but the link condition is not considered. In $[8,9]$ packet scheduling was based on queue information on individual buffer with time varying arrival rate and fixed data rate. A cross-layer scheduling algorithm was presented in [10] using adaptive modulation and coding at the MAC layer for multiple connections with diverse QoS requirement. Similarly in [11] an uplink scheduling algorithm for wireless network with adaptive modulation and coding to quarantee full link utilization and delay requirement for real time application was presented. The work of [11] was for real time and in the uplink, in this paper we present a scheduling algorithm in the downlink for both real time and none real time applications which utilize wireless link condition before scheduling each packet. The term traffic, subscriber station (SS) and service flow will be use interchangeable in this paper. We organized the rest of the paper as follows; Section II focuses on system description, section III deals with proposed algorithm, section IV presents simulation results and conclusions were drawn in section $\mathrm{V}$.

\section{SYSTEM DESCRIPTION}

We developed the system based on Erceg path loss model [1]. We assume the system has one central base station BS, and all subscriber stations are uniformly distributed across the coverage area of the BS. Traffics were admitted based on the system developed in [3] in GPSS mode. All admitted traffics with packet sends during the uplink session; the link condition varies with time 
as the traffics send their packets. The total path loss experienced by the wireless link is a function of distance between the traffics and the BS as well as other environmental factors [12].

If the total path loss of the wireless link is (1), then by [12] the signal to noise ratio in $(\mathrm{dB})$ is obtained using equation (1)

$S N R=\frac{P_{t} \beta_{t} \beta_{r}}{l * R \eta}$

Where $\mathrm{Pt}$ is transmitting power from the $\mathrm{BS}, \beta \mathrm{t}$ is $\mathrm{BS}$ antenna gain, $\beta t$ is ss antenna gain, $\mathrm{R} \eta$ is the receiver sensitivity. The computed SNR value in (1) is used in choosing the required modulation scheme and coding rate for each transmitted packet as shown in table $1[2]$.

TABLE I

RECEIVER SNR IN (DB)

\begin{tabular}{ccc}
\hline \hline $\begin{array}{c}\text { Modulation } \\
\text { type }\end{array}$ & Coding rate & SNR $(\mathrm{dB})$ \\
\hline QPSK & $1 / 2$ & 5.0 \\
& $3 / 4$ & 8.0 \\
16-QAM & $1 / 2$ & 10.5 \\
& $3 / 4$ & 14.0 \\
64-QAM & $1 / 2$ & 16.0 \\
& $2 / 3$ & 18.0 \\
& $3 / 4$ & 20.0 \\
\hline \hline
\end{tabular}

When the modulation scheme is chosen, the required number of slots based on wireless link condition can be obtained [2,3]. The total number of slot per frame depends on the permutation used and DL/UL ratio of the OFDMA on the frame [1]. A slot composes of subchannel in OFDMA symbol, for partial usage subchannelization (PUSC) permutation in the downlink, a slot is one subchannel in two OFDMA symbols [1]. The total physical data rate of a slot is given by equation (2).

$$
\text { slot }=l_{d} * j_{s} * C_{r} * \frac{\log (m)}{v_{d}}
$$

Where ld is the number of OFDMA symbol in one slot, js is the number of subcarriers per slot $\mathrm{Cr}$ is the coding rate $\mathrm{m}$ is modulated symbol and vd is the frame duration in second. From equation 2, the total number of slots required by each packet can be evaluated. A packet with $\mathrm{P}$ bytes requires $\mathrm{PS}$ number of slots given in equation (3) as

$$
P S=\operatorname{ceil}\left(\frac{8 * P}{\operatorname{slot} * v_{d}}\right)
$$

By using equation (1), (2) and (3), each packet transmitted from subscriber station, will have the estimated wireless link condition and hence the scheduler will use this input to schedule the packet with given required QoS. The SNR value estimated at the physical layer is fed to the scheduler in the MAC layer which estimates the number of slots required by the packet and scheduled it. Figure 1 shows the pictorial view of the scheduler and the buffers.

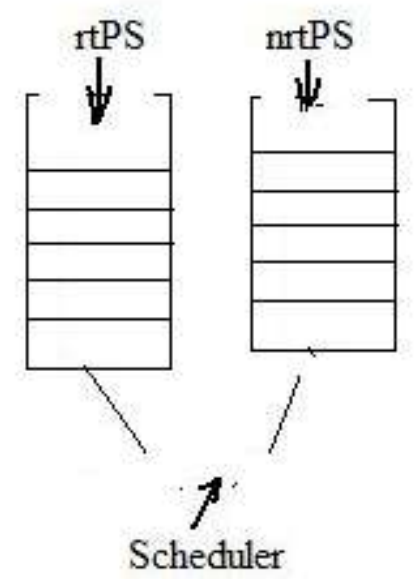

Figure 1 Scheduler for rtPS and nrtPs

Each packet comes with size and SNR, its arrival time is recorded by the BS. The packets are scheduled based on the proposed algorithm.

\section{PROPOSED SCHEDULING ALGORITHM}

It was given in [2] that the packet size in IEEE802.16e is limited to power of two values in the range of 16 to 1024 bytes. Hence, packet size for both rtPs and nrtPS were limited to this range and each transmitted packet size is $2 \mathrm{~N}$ bytes where $\mathrm{N}$ is an integer ranges from 4 to 10. The SNR received for each transmitted packet is a pseudo-random value drawn from a normal distribution with mean $20 \mathrm{~dB}$ and standard deviation $15 \mathrm{~dB}$. The buffer size for both rtPS and nrtPS were set to $\mathrm{k}$ so that each time packet must be removed from the buffer when it's full before the next can be accommodated otherwise the packet is assumed to be lost.

\section{III.1 nrtPs Traffics}

None real time traffics have packets of different size, it required variable bit rate application the packet in nrtPs are scheduled based on their delay and latency. The buffer is visited by the scheduler when the oldest packet in the buffer has reached a preset percentage of its latency. For a packet with the arrival time $t 1$ and the latency $\mathrm{t} 2$ to be scheduled at time $\mathrm{t} 3$, the condition $\mathrm{t} 3-\mathrm{t} 1>=$ preset $(\%) * t 2$ should be satisfied and the packets are scheduled based on the earliest deadline first.

\section{III.2 rtPs Traffics}

Real time traffics are very much sensitive to delay with variable packet size. By default the algorithm always scheduled real time traffics by earliest deadline first manner when the scheduling conditions of none real time has not been met. The packets have variable size each comes with its own SNR.

The approximate algorithm is for selecting the packet to schedule is as follows;

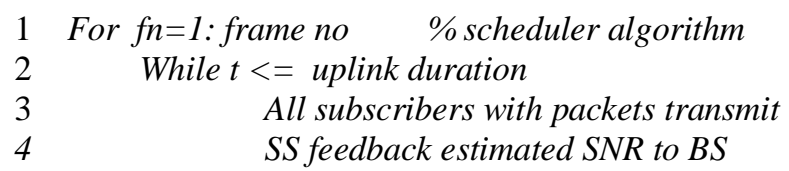




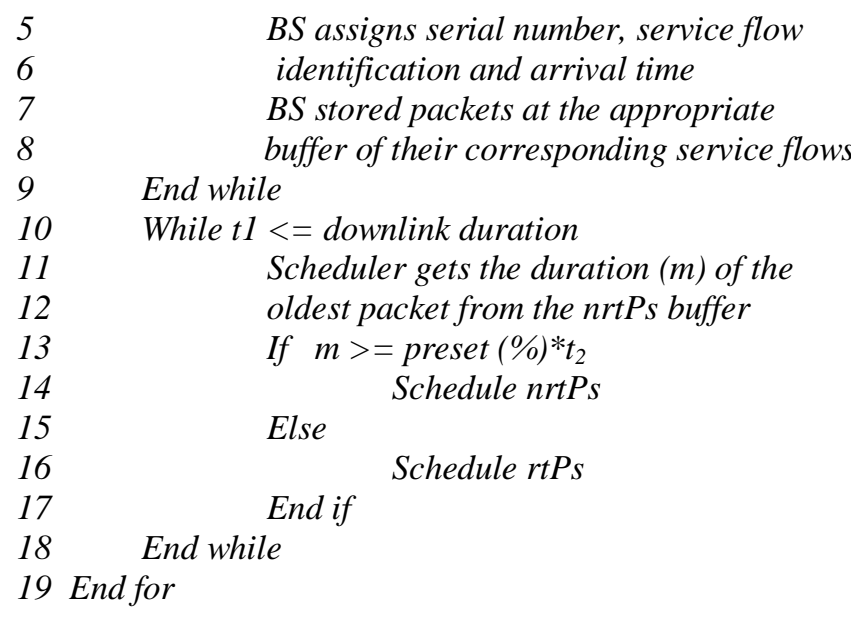

Scheduling Algorithm is how the slots are allocated to the packets on the frame which is part of the algorithm above can be approximated also as follows

0 While $h<=$ frame duration (downlink)

1 For $p=1$ : no of packets \%algorithm on each buffer

2 For the received packets

$3 \quad$ Evaluate the total slots required based on link

4 condition.

5 If frame $=$ new \%(fresh frame no packet on it $)$

$6 \quad$ Scheduled the packet

$7 \quad$ Marked the used slots

$8 \quad$ Next packet

$9 \quad$ Else if frame $=$ used \% ( carrying some packets)

10 If required slots $<=$ Unmarked slots

11

12

13

14

15

16

17

18

19

20

21

End for

Schedule the packets
Marked the used slots
Next packet

Else

Wait for the next new frame

If packet delay >= latency

Drop the packets

Next packets

End if

End if

End while

\section{Simulation Results}

The simulation parameters settings is shown in Table 2, during the uplink session all service flows with packet transmit, we assumed that all the subscriber stations participating has been admitted as in [3]. Base station received all transmitted packets from subscriber stations, assigned packet serial number, packet service flow identification (SFID), arrival time and stores the packet in appropriate buffer of the service flow. Each transmitted packet has its own estimated SNR value, details on the estimated SNR is given in [3].

During the downlink session, the scheduler in the BS scheduled the packets base on the proposed algorithm in section 3. Two parameters (packet size and SNR value) are used to allocate the required number of slots of each packet. If the required number of slots on the current frame is not enough to schedule the current packet, its delay to the next frame provided its latency will not be reached otherwise the packet is loss.

The buffers were used each for different service flow, each buffer can store 500 packets at a time, if the buffer is full and there is packet on the queue, that packet is lost. Each packet scheduled, is removed from the buffer and the memory is declared empty so that it can be used to store the next packet. The uplink duration was set to $5.2 \mathrm{~ms}$ and the downlink is $14.8 \mathrm{~ms}$, the simulation time was set to $20 \mathrm{~s}$ and a total of 1000 frames were used in the scheduling. The simulation results were shown in the Figures below.

Table 2 parameter settings

\begin{tabular}{|l|l|}
\hline Parameter & Settings \\
\hline FFT Size & 512 \\
\hline Bandwidth & $5 \mathrm{MHz}$ \\
\hline DL/UL ratio & $30: 17$ \\
\hline No. of Subchannels & 15 \\
\hline No. of frames & 1000 \\
\hline Frame duration & $20 \mathrm{~ms}$ \\
\hline Slots/frame (downlink) & 225 \\
\hline Permutation & PUSC \\
\hline Buffer size & 500 packets \\
\hline Traffics & rtPS and nrtPS \\
\hline Latency for rtPs & $150 \mathrm{~ms}$ \\
\hline Latency for nrtPs & $800 \mathrm{~ms}$ \\
\hline Preset \% for nrtPs & $80 \%$ \\
\hline Packets sizes & $16,32,64,128,256,512$ and \\
& 1024 byte \\
\hline Modulation scheme & QPSK, 16-QAM, 64-QAM \\
& coding rate $1 / 2,2 / 3$, and $3 / 4$ \\
\hline Simulation time & $20 \mathrm{~s}$ \\
\hline Path loss model & Erceg \\
\hline Transmission frequency & $2.5 \mathrm{GHz}$ \\
\hline Path loss exponents & 4.20 \\
\hline Shadowing component & 8.9 \\
\hline BS transmitting power & $43(\mathrm{dBm})$ \\
\hline BS antenna gain & $16(\mathrm{dBi})$ \\
\hline Receiver sensitivity & $-107.1(\mathrm{dBm})$ \\
\hline Receiver antenna gain & $-1.10(\mathrm{~dB})$ \\
\hline
\end{tabular}

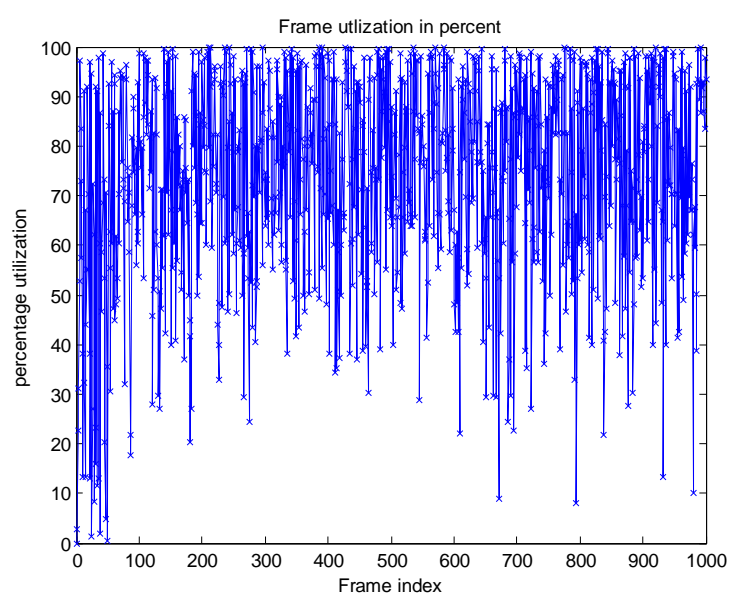

Figure 2 Frame Utilization 


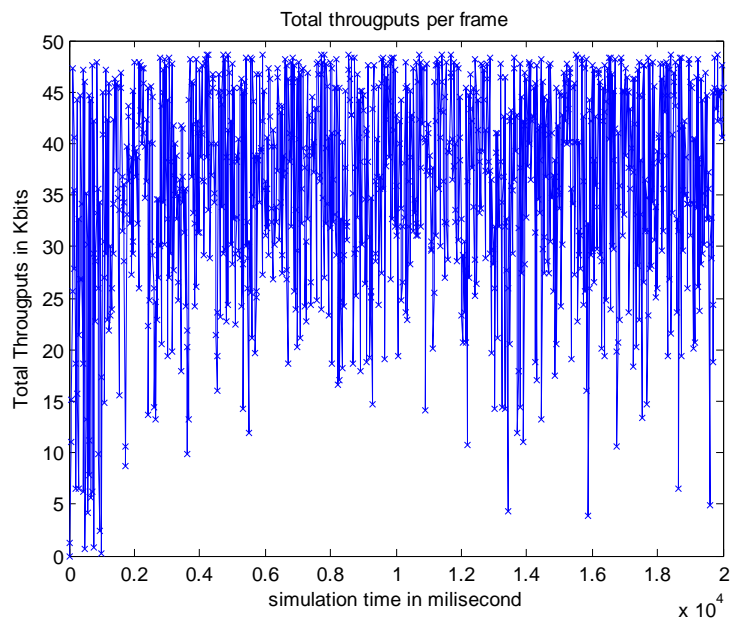

Figure 3 Throughput per unit time

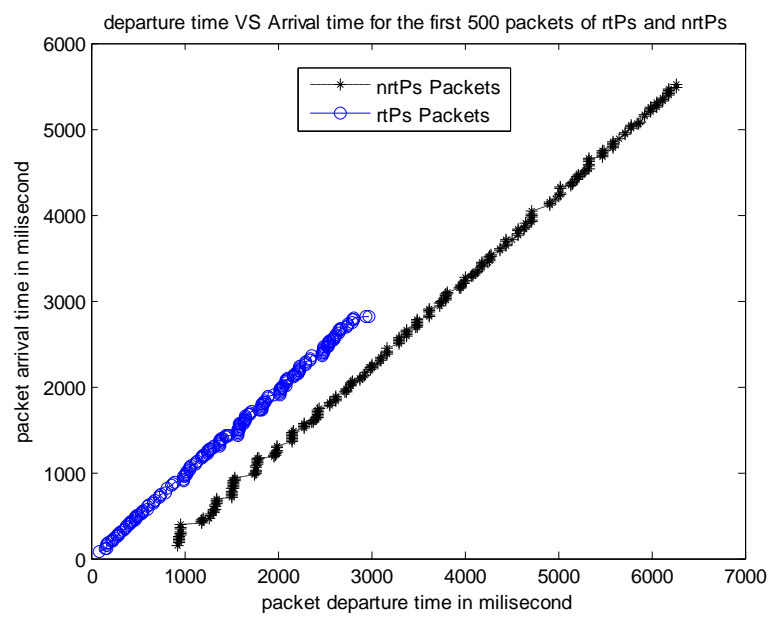

Figure 4 Packets delay in the buffer

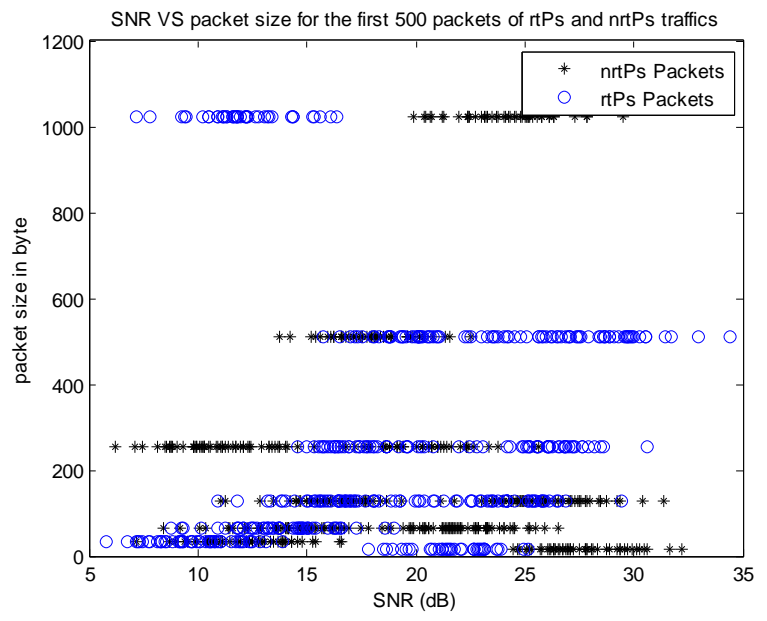

Figure 5 SNR of Each Packet

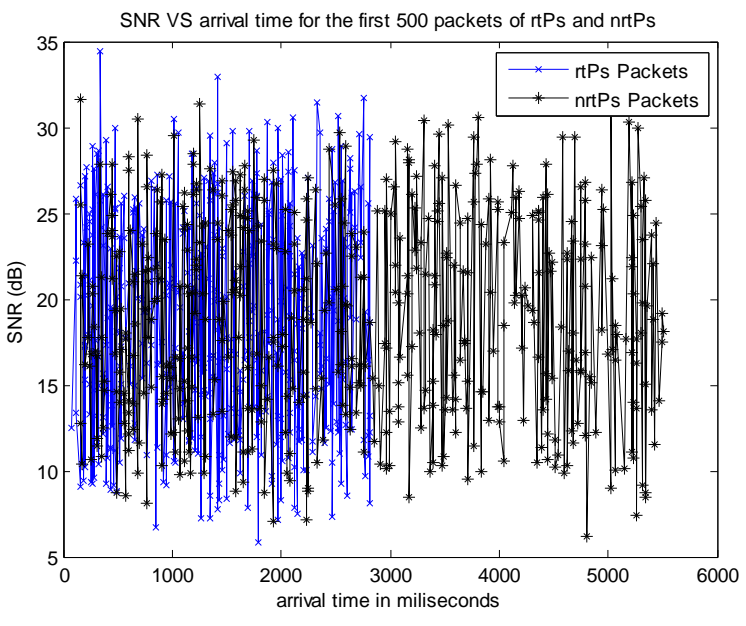

Figure 6 SNR and arrival time

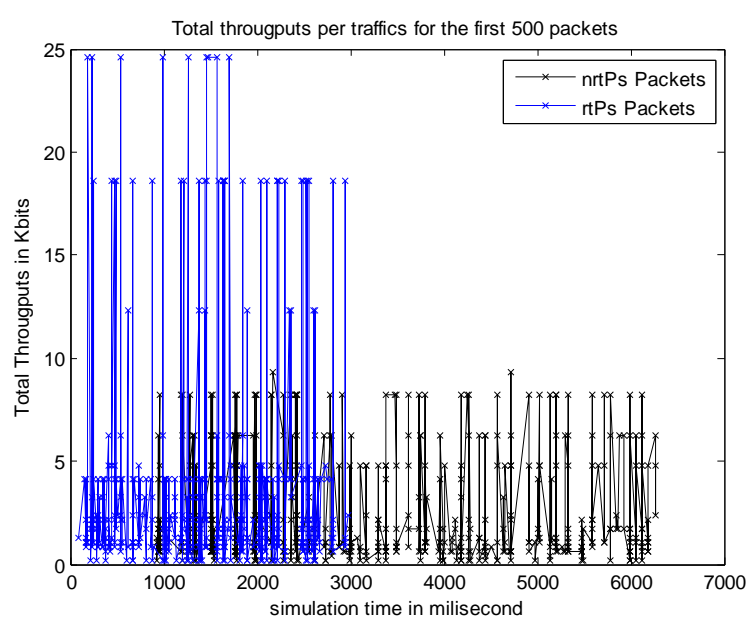

Figure 7 individual traffic throughputs

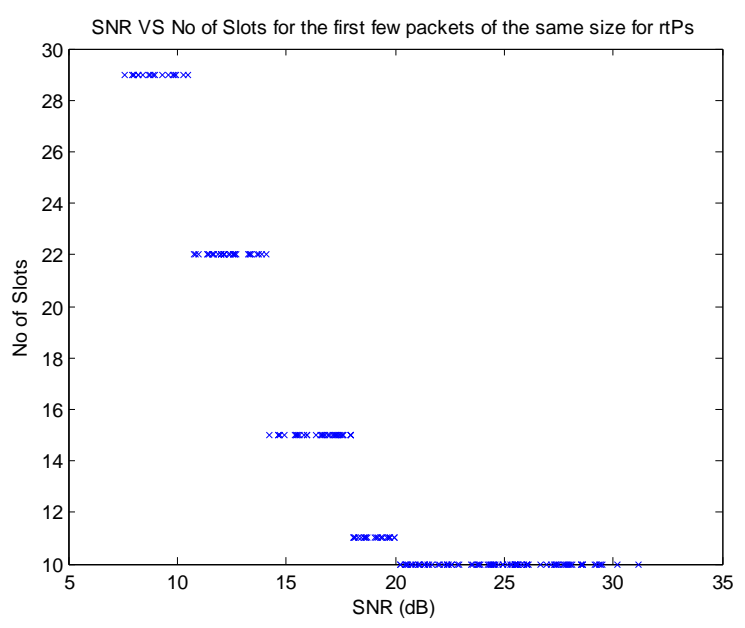

Figure 8 SNR for Fixed Packet size 
Figure 2 shows the percentage utilization of the frame, it can be seen over $80 \%$ of the frame in simulation used more than $90 \%$ of the slots, and each frame carries 225 slots. Figure 3 shows the total throughputs in kilobits as the simulation time varies. Figure 2 and Figure 3 are related since the percentage utilization of the frame depends on the number of slots which determines the throughputs. Packet arriving and departure time is shown in Figure 4, it can be seen that the first 500 packets of rtPs were scheduled within the first $3 \mathrm{~s}$ of the simulation. However for nrtPs, the first 500 packets were scheduled between $780 \mathrm{~ms}$ to $6200 \mathrm{~ms}$. A total of approximately 7000 packets were received and scheduled for both rtPs and nrtPs in every simulation we did.

Figure 5 shows packets sizes and corresponding link condition of both rtPs and nrtPs, the seven different packet size $(16,32,64,128,256,512$ and 1024byte) can be seen to different SNR value. The relation of the SNR value with arrival time is shown in figure 6 , for the two different traffics. The total throughputs of individual service flow of the first 500 packets can be seen in Figure 7. The throughput of both rtPs and nrtPs can be seen to vary in both capacity (amplitude) and time due to continuous changes in the wireless link, since rtPs is a delay sensitive there is fair scheduling among the service flows.

Figure 8 gives the relationships between the selected packets of the same size (256 bytes) from real time traffics and the link condition of the transmitted packet (SNR). It shows that the required number of slots of the packet depends on the link conditions even though the packets have the same size.

Conclusion

In this paper, we presented a slot allocation algorithm for real time and none real time service flow by considering the link condition, we showed that the link condition has important role to play in allocating the required number of slots of given packet. This is very useful in WiMAX wireless network since it employ adaptive modulation and coding.

\section{ACKNOWLEDGMENT}

The authors would like to thank all those who contributed toward making this research successful. Also, we would like to thank to all the reviewers for their insightful comment. This work was sponsored by the Research Management Unit, Universiti Teknologi Malaysia.

\section{REFERENCES}

[1] WiMAX Forum, Krishna Ramadas and Raj Jain, "WiMAX System Evaluation Methodology" version 2.1 July 2008

[2] IEEE Std. 802.16e, "IEEE Standard for local and metropolitan area networks, part 16: Air Interface for Fixed and Mobile Broadband Wireless Access Systems, Amendment 2: Physical and Medium Access Control Layers for Combined Fixed and Mobile Operation in Licensed Band and Corrigendum 1", May 2005

[3] D. S. Shu'aibu, S. K. Syed-Yusof and N. Fisal, "Partition -Based Bandwidth Managements for Mobile WiMAX IEEE802.16e," International Review on Computers and Software (IReCoS) Vol 5 No.4 July 2010.

[4] M. Hawa and D. Petr, "Quality of Service Scheduling in Cable and Broadband Wireless Access Systems," Tenth International Workshop on Quality of Service, May 2002.

[5] JianfengChen, W. Jiao, and H. Wang, "A Service Flow Management Strategy for IEEE 802.16 Broadband Wireless Access Systems in TDD Mode," IEEE, 2005.

[6] Sarat Chandra and Anirudha Sahoo "An Efficient Call Admission Control for IEEE802.16 Networks Proc of the $15^{\text {th }}$ IEEE Workshop on Local and Metropolitan Area Networks, Princeton, New jersey, June 2007. pp 188-193

[7] K. Wongthavarawat and A. Ganz, "Packet scheduling for QoS support in IEEE802.16 broadband wireless access systems", Int Journal of Communication Systems, vol 16, no 1, pp 81-96, Feb 2003.

[8] J.H. Jeon and J.T. Lim, "Dynamic bandwidth allocation for QoS in IEEE802.16 broadband wireless networks," IEICE Trans. Communication vol.E91-B, no 8. Pp 2707-2710, August 2008.

[9] D. Niyato and E. Hossain, "A queuing-theoretic and optimizationbased model for radio resource management in IEEE 802.16 broadband wireless networks," IEEE Trans. Comput, vol 55 no. 11 pp 1473-1488, nov. 2006.

[10] Q. Liu, X. Wang and G. B. Giannakis, “ A Cross layer scheduling algorithm with QoS support in wireless networks, " IEEE Trans. Veh. Technology, vol 55 no. 3 pp 839-847, May 2006.

[11] Hyun-Wook Jo, Jae-Han Jeon and Jong-Tae Lim, "Bandwidth Allocation for QoS Using adaptive Modulation and coding in IEEE 802.16 Networks," IEIEC Trans. Commun, Vol .E92-B, no 12 December 2009.

[12] Bernard Sklar 'Digital Communications Fundementals and Application second edition, Prentice-Hall International Inc Upper Saddle River, New Jersey USA 2001 pg 286-289 